\section{Scientific genius: in different guises}

Dean Keith Simonton's contention that scientific genius is extinct (Nature 493, 602; 2013) may itself be based on an extinct - or at least dying - concept of genius.

In the past, geniuses were lone scientists who distilled extant knowledge to spawn innovation. Simonton lays part of the blame for their extinction on modern team science (see K. Börner et al. Sci. Transl. Med. 2, 49cm24; 2010). But a different kind of genius can exist inside a team. An example might be the member who instinctively optimizes the group's complementary expertise to elicit a ground-breaking discovery.

Even more radical forms of genius are conceivable. The hallmark combination of knowledge and process can create a form of collective genius (A. W. Woolley et al. Science 330, $686-688 ; 2010)$. And as scientists become more connected with each other and technology, genius could emerge from a hybrid of human and machine intelligence.

There is no need to bemoan the demise of the lone genius. Rather, we should be fostering and studying more contemporary forms of scientific genius.

Stephen M. Fiore University of Central Florida, Orlando, Florida, USA.

sfiore@ist.ucf.edu

\section{Scientific genius: will continue to thrive}

Dean Keith Simonton's contention that scientific genius is extinct (Nature 493, 602; 2013) invites comparison with Lord Kelvin's famous speech to the British Association for the Advancement of Science in 1900, in which he remarked, "There is nothing new to be discovered in physics now. All that remains is more and more precise measurement." The discoveries of quantum mechanics and relativity soon made nonsense of this hubristic claim.

Simonton suggests that these were the last new fields, and that disciplines founded since are simply hybrids of existing ones. But science does not proceed in the way followed by the journalist in Charles Dickens's The Pickwick Papers (1836), who, when asked to write on Chinese metaphysics, combined the information he read "for metaphysics under the letter $\mathrm{M}$, and for China under the letter C" from the Encyclopaedia Britannica.

Information theory, for example, founded by Claude Shannon in 1948, is surely a field in its own right — albeit with applications to many different fields. Other emergent fields include network theory and the science of complex systems, which are providing insight into systems from organisms to societies and ecosystems. Opportunities for scientific genius and surprising discoveries are nowhere near exhausted.

Len Fisher University of Bristol, Bristol, UK.

len.fisher@bristol.ac.uk

\section{Don't belittle the rebound effect}

We disagree with Kenneth Gillingham and colleagues' contention that the rebound effect - in which greater consumption offsets the energy saved by increasing efficiency - is exaggerated (Nature 493, 475-476; 2013). We and others have shown rebound effects as large as 60\% (see, for example, M. Frondel et al. Energy Econ. 34, 461-467; 2012).

In our view, energy-efficiency standards are among the least cost-effective ways of lowering carbon emissions (see H. Allcott Am. Econ. Rev. 101, 98-104; 2011). For example, the costs of the US Corporate Average Fuel Economy standard are more than ten times higher than a petroleum tax that induces the same reduction in oil consumption (R. W. Crandall J. Econ. Persp. 6, $171-180 ; 1992)$. This is mainly because of the rebound effect: the standard actually encourages driving by marginally lowering its cost, unlike a tax.

The greater cost-effectiveness of a tax could be undermined by coupling it with an efficiency standard, as Gillingham et al. suggest, because of interaction effects between the two.

Manuel Frondel, Colin Vance Rhineland-Westphalia Institute for Economic Research, Essen, Germany.

vance@rwi-essen.de

\section{Guarding children's genetic privacy}

Your coverage of genetic privacy issues (Nature 493, 451 and Nature 494, 7; 2013) does not address concerns relating to DNA collected from children, who have contributed thousands of biobank samples worldwide.

Open sharing of genotype and phenotype information is crucial for advancing research into childhood diseases, but depends on consent from patients or their parents. Children cannot themselves consent to participation in research with long-term privacy implications. However, parental consent is currently founded on unrealistic promises of anonymity and confidentiality.

For the responsible conduct of research, parents need to be made fully aware of the risk of identifiability before granting consent on their children's behalf (J. E. Lunshof et al. Nature Rev. Genet. 9, 406-411; 2008).

Stringent research protocols are necessary to protect child donors (D. Gurwitz et al. Science 325, 818-819; 2009). But we should not prohibit the publication of sequencing information that could save lives. For example, mutations in calmodulin gene sequences are associated with some sudden infant deaths (L. Crotti et al. Circulation http:// doi.org/kjz; 2013). Jeantine E. Lunshof Harvard Medical School, Boston, Massachusetts, USA. jelunshof@genetics.med.harvard. edu

David Gurwitz Tel Aviv

University, Israel.

\section{'Antifragility' as a mathematical idea}

In his review of my book Antifragile, Michael Shermer mischaracterizes the concept of 'antifragility' (Nature 491, 523; 2012).

'Fragility' can be defined as an accelerating sensitivity to a harmful stressor: this response plots as a concave curve and mathematically culminates in more harm than benefit from random events. 'Antifragility' is the opposite, producing a convex response that leads to more benefit than harm.

We do not need to know the history and statistics of an item to measure its fragility or antifragility, or to be able to predict rare and random ('black swan') events. All we need is to be able to assess whether the item is accelerating towards harm or benefit. The relation of fragility, convexity and sensitivity to disorder is thus mathematical (N. N. Taleb and R. Douady Quant. Finance, in the press) and not derived from empirical data, as Shermer implies.

Shermer's suggestion that I should offer "a checklist of things companies or countries can do to prepare for black-swan events" overlooks 50 or so such heuristics based on the identification of convex responses.

Nassim N. Taleb Polytechnic Institute of New York University, New York, USA.

ntaleb@poly.edu

\section{CLARIFICATION}

The Correspondence headline 'Avoid constructing wind farms on peat' (J. Smith et al. Nature 489, 33; 2012) is misleading: it is developments on non-degraded, pristine peats that should be avoided. 